\title{
|||||||||||||||||||||||||||||||||||||||||||||||||||||||||||||||||||.
}

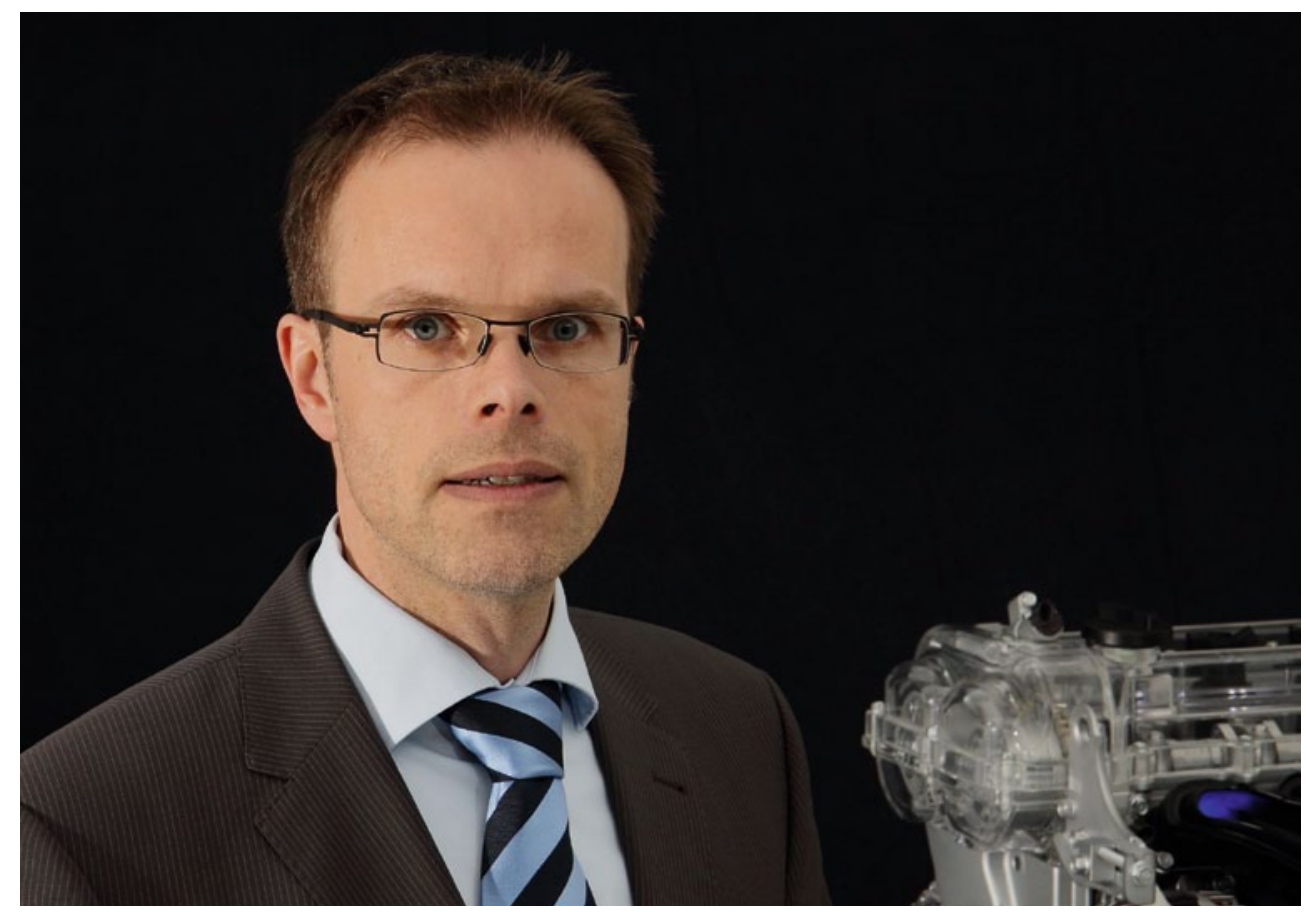

DR.-ING. MICHAEL WINKLER

Manager Powertrain

Hyundai Motor Europe

Technical Center

\section{BEZAHLBARE OTTOMOTOREN FÜR 2020}

Der moderne - zumeist aufgeladene - Ottomotor ist mittlerweile durch eine Diversifizierung der Technik gekennzeichnet. Es finden sich zunehmend variable Ventiltriebe, zwei oder mehrstufige Hubumschaltungen auf der Ein- beziehungsweise auf der Auslassseite oder auch die Zylinderabschaltung selbst bei kleinen Zylinderzahlen. Bei den Einspritzsystemen sind sowohl Saugrohr- sowie Direkteinspritzung mit seitlicher oder zentraler Düsenlage als auch eine Kombination beider Einspritzsysteme mit Magnet- aber auch Piezoinjektoren in Anwendung. Darüber hinaus ist eine Elektrifizierung der Zusatzaggregate wie kennfeldgesteuertes Thermostat, elektrische Wasser-, Ölbeziehungsweise Unterdruckpumpe zu beobachten. Auch der Atkinson-Cycle in Kombination mit Hybridisierung oder Aufladung ist in Serienanwendungen bereits umgesetzt. Der Blumenstrauß der verfügbaren Technologien ist somit groß und bunt.

War die Motorenentwicklung bisher maßgeblich durch eine gewünschte Leistungscharakteristik des Antriebsstrangs sowie durch die Erfüllung von $\mathrm{CO}_{2}$-Flottenverbräuchen geprägt, steht nun die Einführung eines weiteren Grenzwerts für die Partikelanzahl bei Ottomotoren in Europa unmittelbar bevor. Aus technischer Sicht kann dieser Grenzwert innermotorisch durch ein optimiertes Brennverfahren oder durch Abgasnachbehandlung beherrscht werden. Ein auf Partikelanzahl optimiertes Brennverfahren stellt dabei auch beim Ottomotor neue Anforderungen an das Einspritzsystem mit stabilen Kleinstmengen während des Kat-Heizens sowie Mehrfacheinspritzung in Kombination mit gestiegenen Einspritzdrücken. Mittels innermotorischer Maßnahmen kann der Partikelanzahlgrenzwert 6 el1 \#/km für einzelne Fahrzeuge bereits deutlich unterschritten werden.

Die eigentliche Herausforderung ist nun, diesen Wert über die Fahrzeuglebensdauer sicherzustellen. Die dafür notwendigen Kalibriermaßnahmen sowie die Erhöhung des Kraftstoffdrucks im Teillastbereich stehen im Zielkonflikt mit der Verschlechterung des Kraftstoffverbrauchs, wie dies auch schon bei der Einführung des Dreiwege-Katalysators zu beobachten war. Dieser etwaige Mehrverbrauch muss insbesondere mit Blick auf den ab 2020 geltenden $\mathrm{CO}_{2}$-Flottenverbrauch von $95 \mathrm{~g} / \mathrm{km}$ überkompensiert werden. Die dafür einzusetzende Technologie wird sich von Hersteller zu Hersteller unterscheiden. Während insbesondere Premiumhersteller Technologien als Alleinstellungsmerkmal nutzen können, werden Volumenhersteller wie die Hyundai Group neben den Kosten der Technologie auch einen Fokus auf die weltweite Einsatzfähigkeit legen. Die Herausforderung an die Hersteller, technisch und Branding-relevante Lösungskombinationen zu definieren und mit vertretbaren Kosten weltweit $\mathrm{zu}$ vertreiben, wird nicht zuletzt durch die $\mathrm{CO}_{2}$-Strafzahlungen ab 2020 in Europa zusätzlich verschärft.

Die Etablierung neuer Technologien kann deshalb nur gelingen, wenn ein zusätzlicher Kundennutzen in Form von Fahrspaß aber auch Verbrauchsvorteilen im realen Kundenbetrieb erreicht wird und der Kostenvorteil des Ottomotors zum Dieselmotor erhalten bleibt. 